\title{
Prognostic relevance of symptoms versus objective evidence of coronary artery disease in diabetic patients
}

\author{
Michael J. Zellweger ${ }^{a, b, c}$, Rory Hachamovitch ${ }^{a, b}$, Xingping Kang ${ }^{a, b}$, \\ Sean W. Hayes ${ }^{a, b}$, John D. Friedman ${ }^{a, b}$, Guido Germano ${ }^{a, b}$, \\ Matthias E. Pfisterer ${ }^{\mathrm{c}}$, Daniel S. Berman ${ }^{\mathrm{a}, \mathrm{b}, *}$ \\ a Departments of Imaging (Division of Nuclear Medicine) and Medicine (Division of Cardiology), Cedars-Sinai \\ Medical Center, Room A042, 8700 Beverly Blvd., Los Angeles, CA 90048, USA \\ b Department of Medicine, UCLA School of Medicine, Los Angeles, CA, USA \\ c Department of Cardiology, University Hospital, Basel, Switzerland
}

Received 24 June 2003; revised 4 February 2004; accepted 13 February 2004

\section{KEYWORDS}

Diabetes mellitus;

Silent ischaemia;

Myocardial perfusion SPECT;

Outcome;

Prognosis

\begin{abstract}
Aim Little is known about the prognostic significance of silent versus symptomatic coronary artery disease (CAD) in diabetic patients. We therefore assessed the incidence of scintigraphic evidence of CAD in diabetic patients without known CAD and the impact of symptoms and scintigraphic findings on prognosis.

Methods and results A consecutive series of 1737 diabetic patients without known CAD underwent dual-isotope myocardial perfusion SPECT (MPS) and 1430 were followed-up for a median of $2(1-8.5)$ years. Critical events were defined as myocardial infarction or cardiac death.

Objective evidence of CAD was found in $39 \%$ of 826 asymptomatic diabetic patients, in $51 \%$ of 151 diabetic patients with shortness of breath (SOB), and in $44 \%$ of 760 diabetic patients with angina. During follow-up, 98 critical events occurred. Annual critical event rates were $2.2 \%$ in asymptomatic, $3.2 \%$ in angina, and $7.7 \%$ in diabetic patients with shortness of breath ( $p<0.001$ versus other groups). With MPS evidence of CAD, critical event rates increased to 3.4\% (asymptomatic), 5.6\% (angina), and $13.2 \%$ (SOB) $(p \leqslant 0.009$ versus no evidence of CAD). Age, hypertension, shortness of breath, scarring and ischaemia were independent predictors of critical events. MPS findings added incremental information to prescan information regarding outcome prediction.

Conclusions In asymptomatic diabetic patients, the rate of objective evidence of CAD and annual critical events were similar to those found in diabetic patients with angina. The outcome was three times worse in diabetic patients with shortness of breath. MPS findings were strongly predictive of outcome and proved valuable for risk stratification.
\end{abstract}

(C) 2004 Published by Elsevier Ltd on behalf of The European Society of Cardiology.

\footnotetext{
Supported in part by grants from Bristol Myers Squibb Medical Imaging and Fujisawa Healthcare, Inc. This work was presented in part at the Annual Meeting of the European Society of Cardiology 2003 in Vienna.

* Corresponding author. Tel.: +1-310-423-4216/4223; fax: +1-310-4238396/0811.

E-mail address: daniel.berman@cshs.org (D.S. Berman).
} 


\section{List of Abbreviations}

$\begin{array}{ll}\text { CAD: } \text { Coronary artery disease } & \text { SRS: Summed rest score } \\ \text { SOB: Shortness of breath } & \text { SDS: Summed difference score } \\ \text { MPS: Myocardial perfusion SPECT } & \text { NRS: Number of nonreversible segments }\end{array}$

SSS: Summed stress score

\section{Background}

The prevalence of type II diabetes is increasing rapidly in Western nations due to the aging of the population, increased frequency of obesity, and suboptimal nutritional habits. ${ }^{1,2}$ Type II diabetes is associated with a substantially elevated risk of cardiovascular morbidity and mortality. This adds to the prospect that an "epiepidemic" of coronary artery disease (CAD) will appear over the coming decades. Atherosclerosis accounts for $65-80 \%$ of all deaths among diabetic patients, compared with one-third of all deaths in the general population. $^{3}$ The cardiovascular mortality rate is more than twice as high in diabetic men and more than fourfold greater in diabetic women, compared with their nondiabetic counterparts. ${ }^{4}$ When advanced CAD is detected in diabetics, coronary artery bypass surgery has been shown to improve survival and may be superior to percutaneous coronary interventions, probably due in part to the presence of more diffuse CAD in diabetic patients. ${ }^{5}$ However, due to the high prevalence of silent CAD in diabetics, ${ }^{6-10}$ up to $65 \%$, the detection of $C A D$ deserving intervention is crucial.

Myocardial perfusion SPECT (MPS) is widely used in the risk stratification of patients with known or suspected CAD. ${ }^{11-19}$ However, little is known about the interrelationship of symptoms and MPS findings in diabetic patients. Clinically, this is particularly important since symptoms in diabetic patients are more often absent or atypical (shortness of breath) than in nondiabetic patients. $8,20,21$ The aim of the present study was therefore twofold: (1) to assess the incidence of MPS evidence of $C A D$ in a diabetic patient cohort without known CAD, (2) to evaluate the impact of patient symptoms and MPS findings on prognosis in such diabetic patients.

\section{Methods}

\section{Study population}

A large consecutive cohort of 2943 diabetic patients referred for CAD evaluation were tested by exercise or adenosine stress MPS between February 1991 and May 1998. The diagnosis of diabetes mellitus was based on a known history of glucose intolerance and/or antidiabetic drug therapy at the time of stress testing, but no details were recorded in the case report form. Patients with a history of CAD (history of myocardial infarction or revascularisation) were excluded, leaving 1737 patients (59\% of the initial cohort) as the diagnostic study population. Of these, $760(44 \%)$ had chronic chest pain (angina pectoris or angina-like pain) and served as the control group, whereas 977 patients $(56 \%)$ had either shortness of breath (SOB; $n=151)$ only or no specific cardiac complaints $(n=826)$. The median follow-up time for these patients was 2 years (all $>1$ year, range $1-8.5$ years, interquartile range 1.6-2.6 years). Not included in this follow-up evaluation were 161 patients who underwent revascularisation as a direct consequence of the MPS findings $(<60$ days after MPS) $)^{22,23}$ and 146 patients who were lost to follow-up (8\%), leaving a prognostic study population of 1430 patients.

\section{Imaging and stress protocol}

All patients underwent rest TL-201/stress Tc-99m sestamibi MPS, as previously described. ${ }^{24}$ Patients were injected intravenously at rest with Tl-201 $(2.5-3.5 \mathrm{mCi})$, with the dose adjusted for patient weight. Rest TL-201 SPECT was initiated 10 min after injection of the radionuclide.

\section{Exercise MPS protocol}

Immediately following imaging, patients performed a symptomlimited treadmill exercise test using standard protocols, with 12-lead electrocardiographic recording each minute of exercise and continuous monitoring of leads AVF, V1, and V5. Exercise endpoints included physical exhaustion, severe angina, sustained ventricular tachycardia, haemodynamically significant supraventricular arrhythmias, or exertional hypotension. At near maximal stress, a $20-30 \mathrm{mCi}$ dose of Tc-99m sestamibi was injected (the actual patient dose was adjusted for patient weight) and exercise continued for 1 additional minute after injection. Tc-99m sestamibi SPECT imaging was begun $15-30$ min later. ${ }^{24}$

\section{Adenosine MPS protocol}

Patients were instructed not to consume caffeine-containing products for $24 \mathrm{~h}$ before MPS. Following rest TL-201 SPECT, pharmacologic stress was performed using adenosine infusion $(140 \mu \mathrm{g} / \mathrm{kg} / \mathrm{min}$ for 5-6 $\mathrm{min})$. Tc-99m sestamibi $(20-30 \mathrm{mCi})$ was injected at the end of the $2-3$ min of infusion and SPECT was initiated $60 \mathrm{~min}$ after the end of adenosine infusion. ${ }^{24}$ For patients who underwent exercise as an adjunct to adenosine infusion, low-level treadmill exercise was performed at $0 \%$ and 1-1.7 mph.

During both types of stress, blood pressure was measured and recorded at rest, at the end of each exercise stage, and at peak exercise. Maximal ST-segment change at $80 \mathrm{~ms}$ after the $\mathrm{J}$ point was assessed as horizontal, upsloping, or downsloping.

\section{SPECT acquisition protocol}

SPECT was performed as previously described using circular or elliptical $180^{\circ}$ acquisition for 64 projections, $20 \mathrm{~s}$ per projection. ${ }^{24}$ During imaging, two energy windows for Tl-201 were utilised (30\% window centred on the $68-80 \mathrm{keV}$ peak and a $10 \%$ window centred on the $167 \mathrm{keV}$ peak) and a 15\% window centred on the $140 \mathrm{keV}$ peak was used for Tc-99m sestamibi. No attenuation or scatter correction was used. 


\section{Image interpretation}

Semiquantitative visual interpretation was performed using a 20 -segment model as previously described. ${ }^{25}$ Each segment was scored using a 5 -point scoring system $(0=$ normal, $1=$ equivocal, $2=$ moderate, 3 = severe reduction of radioisotope uptake, and $4=$ apparent absence of detectable tracer uptake in a segment). A summed stress score (SSS) was obtained by adding the scores of the 20 segments of the stress images. ${ }^{24} \mathrm{~A}$ summed rest score (SRS = extent of scar) was obtained in a similar way by adding the scores of the 20 segments on the rest images. Subsequently, to assess the extent of ischaemia, a summed difference score (SDS = extent of ischaemia) was calculated by subtracting the SRS from the SSS. The number of nonreversible segments (NRS) (stress-rest score combinations of $4-4,4-3,3-3,3-2$, or $2-2$ ) was used as a surrogate marker of left ventricular ejection fraction (with each NRS representing approximately $5 \%$ of the myocardium), since ejection fraction data from gated SPECT were not available for the entire patient population.

As per our previous work, SSS $<4$ was considered normal; 4-8, mildly abnormal; 9-13 moderately abnormal, and $>13$ severely abnormal. ${ }^{13}$ Similarly, patients with SDS $<2$ and $\geqslant 2$ were considered nonischaemic and ischaemic, respectively.

\section{Patient follow-up}

Individuals who were unaware of the patients' test results performed follow-up using scripted telephone interviews. The endpoint of interest was hard events: myocardial infarction or cardiac death. Cardiac death was defined as death due to any cardiovascular cause and was confirmed by review of the death certificate, hospital chart, or physician's record; nonfatal myocardial infarction was documented by a consistent history accompanied by elevation of cardiac enzymes and/or new $Q$ waves on the ECG. ${ }^{11,25}$

\section{Statistical analysis}

Comparisons between patient groups were performed using oneway ANOVA (followed by the Bonferroni test) for continuous variables and $\mathrm{a} \chi^{2}$ test for the categorical variables. When the categorical variables of three patient groups were compared, the groups were tested pairwise, followed by the Bonferroni test. All continuous variables are described as means \pm SD. A $p$ value $<0.05$ was considered statistically significant.

Predicted event rates were determined using each patient's hazard scores from the final Cox proportional hazards model. These event rates were annualised by dividing them by the mean follow-up time. Kaplan-Meier cumulative event-free survival curves were used to compare the survival of different patient groups (log-rank test).

Regarding prognosis, univariate predictors of critical events were evaluated by a Cox proportional hazards model. The association between relevant variables and risk of adverse outcomes was determined using a multivariate Cox proportional hazards model. The selection of the variables entered in the model was based on univariate significance (age, hypertension, shortness of breath, stress modality, SRS, SDS), and reported prognostic indicators such as sex, hypercholesterolaemia, smoking, family history of $C A D$, angina, angina during stress testing, and ECG response during stress testing. A forward stepwise method was then applied using a $p$ value $<0.10$ for inclusion and $>0.20$ for exclusion into the model.
Since the patient population that had a left ventricular ejection fraction available was too small to run a separate analysis with the endpoint of critical event, a separate model was built incorporating NRS instead of SRS (all other covariates unchanged).

The incremental value of nuclear testing was determined by calculating the change in global $\chi^{2}$ after adding the nuclear variables (SRS, SDS) to the prescan information (age, sex, shortness of breath, angina, hypertension, hypercholesterolaemia, smoking, family history of CAD, stress modality, angina during stress testing, and ischaemic ECG during stress testing) with respect to prognosis (Cox model). Analyses were made using SPSS statistical package (version 11.5).

\section{Results}

\section{Baseline characteristics in relation to symptoms}

Of 1737 diabetic patients referred for evaluation of CAD, $826(47 \%)$ were asymptomatic and $151(9 \%)$ had shortness of breath. The characteristics of these patients in relation to the control group of 760 patients with chronic angina (44\%) are shown in Table 1 . Asymptomatic patients were more likely to be male, had a family history of CAD less often, and had a lower extent of ischaemia (SDS) than symptomatic patients and differed from patients with shortness of breath in lower age, smaller percentage of female patients, higher frequency of exercise treadmill testing and ischaemic ECG changes, and lower overall abnormality of MPS findings (SSS), scarring (SRS) and extent of ischaemia (SDS) (Table 1). Patients with shortness of breath were older than the other two patient groups, more often had evidence of scarring and larger scars (SRS, NRS) than asymptomatic patients and patients with angina, a higher rate of scarring and ischaemia with more overall abnormality of MPS findings (SSS) and only slightly greater extent of ischaemia (SDS) (Table 1). In addition, patients with shortness of breath could perform an exercise treadmill test less often and had ischaemic ECG changes less often (Table 1).

Patients with shortness of breath had a higher number of nonreversible segments compared to the other two patient groups (Table 1). Together with the lower left ventricular ejection fraction observed in a subgroup analysis of 388 patients $(27 \%)$ for which this data was available (patients with shortness of breath had a significantly lower ejection fraction $[52 \pm 19 \%, p \leqslant 0.025$ versus the other two groups] than asymptomatic patients [58 $\pm 14 \%]$ and patients with angina $[60 \pm 13 \%])$, this points to a higher rate of silent prior myocardial infarction in patients with shortness of breath.

Of note, the overall MPS abnormality, expressed as SSS, was not significantly different between patients with $(6.0 \pm 8.5)$ and without angina (5.5 \pm 7.7$)$.

\section{MPS evidence of CAD in relation to symptoms}

Overall, 730 (42\%) patients had abnormal MPS results. There were no significant differences between the asymptomatic group (39\%) and patients with angina 
Table 1 Patient characteristics by symptomatic status

\begin{tabular}{lllll}
\hline & All patients $(n=1737)$ & Asymptomatic $(n=826)$ & SOB $(n=151)$ & Angina $(n=760)$ \\
\hline Age (years) & $65.8 \pm 11.5$ & $65.0 \pm 11.6^{\dagger}$ & $71.5 \pm 10.1$ & $65.4 \pm 11.3^{\#}$ \\
Female sex, $n$ (\%) & $848(49)$ & $351(43)^{*, \dagger}$ & $81(54)$ & $416(55)$ \\
Hypercholesterolaemia, $n(\%)$ & $824(47)$ & $374(45)$ & $64(42)$ & $386(51)$ \\
Smoker, $n$ (\%) & $216(12)$ & $93(11)$ & $23(15)$ & $100(13)$ \\
Hypertension, $n$ (\%) & $1140(66)$ & $520(63)$ & $105(70)$ & $515(68)$ \\
Family history of CAD, $n(\%)$ & $357(21)$ & $140(17)^{*}$ & $32(21)$ & $185(24)$ \\
Exercise treadmill test, $n(\%)$ & $893(51)$ & $443(54)^{\dagger}$ & $48(32)$ & $402(53)^{\#}$ \\
Ischaemic ECG, $n$ (\%) & $270(16)$ & $127(15)^{\dagger}$ & $9(6)$ & $134(18)^{\#}$ \\
SSS & $5.8 \pm 8.4$ & $5.0 \pm 7.7^{\dagger}$ & $8.8 \pm 11.0$ & $6.0 \pm 8.5^{\#}$ \\
SRS & $1.2 \pm 4.0$ & $1.1 \pm 3.8^{\dagger}$ & $3.3 \pm 7.1$ & $0.9 \pm 3.2^{\#}$ \\
SDS & $4.6 \pm 7.0$ & $3.9 \pm 6.4^{*}, \dagger^{\dagger}$ & $5.5 \pm 8.0$ & $5.1 \pm 7.5$ \\
NRS & $0.5 \pm 1.5$ & $0.4 \pm 1.4^{\dagger}$ & $1.3 \pm 2.6$ & $0.4 \pm 1.3^{\#}$ \\
Pts with normal MPS, $n$ (\%) & $1007(58)$ & $504(61)^{\dagger}$ & $74(49)$ & $429(56)$ \\
Pts with scarring, $n$ (\%) & $66(4)$ & $36(4)$ & $10(7)$ & $20(3)^{\#}$ \\
Pts with ischaemia, $n$ (\%) & $465(27)$ & $203(25)$ & $31(20)$ & $231(30)^{\#}$ \\
Pts with scarring plus ischaemia, $n(\%)$ & $199(11)$ & $83(10)^{\dagger}$ & $36(24)$ & $80(11)^{\#}$
\end{tabular}

SOB, shortness of breath; SSS, summed stress score (overall abnormality of MPS); SRS, summed rest score (scarring); SDS, summed difference score (extent of ischemia); NRS, number of nonreversible segments.

" Significant difference between patients with angina and patients with shortness of breath (significant difference: $p<0.02$ ).

Significant difference between asymptomatic patients and patients with shortness of breath (significant difference: $p<0.02$ ).

"Significant difference between asymptomatic patients and patients with angina (significant difference: $p<0.02$ ).

(44\%). The highest rate of abnormal MPS results was in patients with shortness of breath $(51 \%)$, being significantly higher than in asymptomatic patients (39\%, $p=0.006)$, probably because more patients with shortness of breath had evidence of scarring (prior silent myocardial infarction) with or without ischaemia compared to the other two groups (Table 1). Thus, in the low-risk group of diabetic patients tested, more than one-third had MPS evidence of CAD, irrespective of symptomatic status.

It is noteworthy that the 307 patients excluded from the prognostic part of the study had ischaemic ECG changes during stress testing more often, a higher overall abnormality of MPS findings (SSS), more extensive scarring (SRS), and more extensive ischaemia (SDS) than the prognostic study population $(n=1430)$ (data not shown). This is consistent with the fact that 161 of these patients underwent early revascularisation.

\section{Outcome events and event prediction}

During a median follow-up of 2 years (range 1-8.5 years), 98 critical events occurred in the prognostic patient population of 1430 patients, which is consistent with an annual event rate of $3.1 \%$ : $65 \%$ were nonfatal myocardial infarctions and $35 \%$, cardiac deaths. Annual event rates were similar in females and males, $3.3 \%$ and $2.9 \%$, respectively $(p=0.66)$. Asymptomatic patients, patients with angina, and patients with shortness of breath had annual critical event rates of $2.2 \%, 3.2 \%$, and $7.7 \%$, respectively. Patients with shortness of breath had significantly more events than asymptomatic patients $(p<0.0001)$ and patients with angina $(p=0.0002)$, whereas the difference between asymptomatic patients and patients with angina was statistically nonsignificant $(p=0.44)$.

\section{Event-free survival as a function of MPS results}

Overall, patients with normal MPS results had a significantly lower annual critical event rate than patients with abnormal MPS results, $1.9 \%$ and $5.4 \%$, respectively ( $p<0.0001)$; this held true for all three patient groups (Fig. 1). In patients with normal MPS results, annual critical event rates were not significantly different when asymptomatic patients, patients with angina, and patients with shortness of breath were compared. In contrast, in patients with abnormal MPS findings, patients with shortness of breath had significantly higher annual critical event rates than asymptomatic patients $(p<0.0001)$ and patients with angina $(p=0.0008)$, whereas asymptomatic patients and patients with angina had a similar outcome $(p=0.33)$. The cumulative eventfree survival of patients with abnormal MPS results is shown in Fig. 2. Annual event rates of patients with normal MPS findings and evidence of scarring, of ischaemia, and of scarring plus ischaemia are shown in Table 2. Annual event rates were lowest in patients with normal MPS results and highest in patients with evidence of scarring plus ischaemia.

\section{Univariate and multivariate predictors of critical events}

Univariate predictors of critical events are summarised in Table 3. Age, hypertension, shortness of breath, phar- 


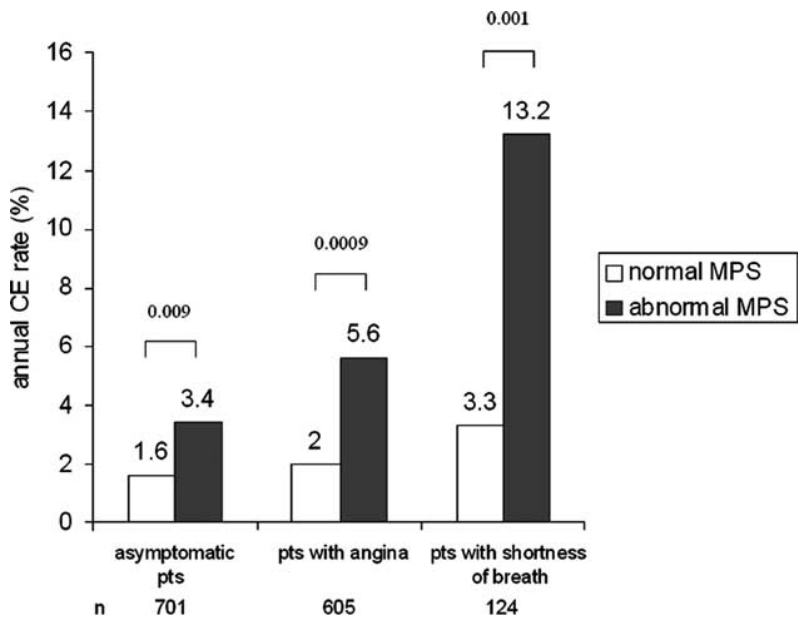

Fig. 1 Annual event rates as a function of MPS results and symptomatic status $(n=1430)$. Pts, patients; MPS, myocardial perfusion SPECT.

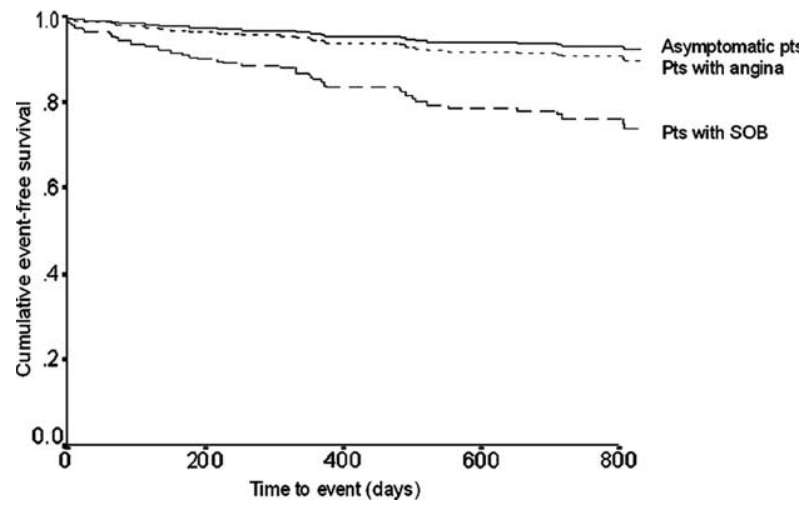

Fig. 2 Kaplan-Meier event-free survival curves as a function of symptoms in patients with abnormal MPS. Asymptomatic patients and patients with angina had a significantly better survival than patients with SOB $(p<0.001),(n=1430)$. Pts, patients; SOB, shortness of breath.

Table 2 Annual critical events rate as a function of MPS results and symptoms

\begin{tabular}{lllll}
\hline & All patients $(n=1430)$ & Asymptomatic $(n=701)$ & SOB $(n=124)$ & Angina $(n=605)$ \\
\hline Pts with normal MPS & $1.9 \%$ & $1.6 \%$ & $3.3 \%$ & $2.0 \%$ \\
Pts with scarring & $4.1 \%$ & $4.7 \%$ & $5.1 \%$ & $2.7 \%$ \\
Pts with ischaemia & $4.6 \%$ & $2.9 \%^{\dagger}$ & $13.6 \%$ & $5.2 \%$ \\
Pts with scarring plus ischaemia & $7.6 \%$ & $4.0 \%^{\dagger}$ & $15.7 \%$ & $7.6 \%$ \\
\hline
\end{tabular}

"Significant difference between patients with angina and patients with shortness of breath (significant difference: $p<0.02$ ).

† Significant difference between asymptomatic patients and patients with shortness of breath (significant difference: $p<0.02$ ).

Table 3 Univariate predictors of critical events

\begin{tabular}{lllllc}
\hline & Hazard ratio & $\chi^{2}$ & \multicolumn{2}{c}{$95 \%$ confidence interval } & $p$ \\
\cline { 3 - 4 } & & & Lower & Upper \\
\hline Age & 1.05 & 22.1 & 1.03 & 1.07 & $<0.0001$ \\
Female sex & 0.9 & 0.2 & 0.6 & 1.4 & 0.66 \\
Hypertension & 1.6 & 4.3 & 1.03 & 2.59 & 0.04 \\
Smoking & 1.1 & 0.03 & 0.6 & 1.9 & 0.87 \\
Hypercholesterolaemia & 1.3 & 1.9 & 0.9 & 2.0 & 0.17 \\
Family history of CAD & 1.3 & 1.1 & 0.8 & 2.0 & 0.29 \\
Angina & 1.1 & 0.3 & 0.7 & 1.7 & 0.59 \\
Shortness of breath & 2.9 & 18.5 & 1.8 & 4.7 & $<0.0001$ \\
Pharmacologic stress & 2.1 & 11.5 & 1.4 & 3.2 & 0.001 \\
Angina during stress & 1.1 & 0.1 & 0.5 & 2.5 & 0.74 \\
ECG ischaemic during test & 1.2 & 0.4 & 0.7 & 1.09 & 0.52 \\
SSS & 1.07 & 51.1 & 1.05 & 1.1 & $<0.0001$ \\
SRS & 1.07 & 21.9 & 1.04 & 1.09 & $<0.0001$ \\
SDS & 1.07 & 34.1 & 1.04 & 1.28 & $<0.0001$ \\
NRS & 1.19 & 19.4 & 1.10 & $<0.0001$ \\
\hline
\end{tabular}

CE, critical event (myocardial infarction or cardiac death); SSS, summed stress score; SRS, summed rest score; SDS, summed difference score; NRS, number of nonreversible segments.

macologic stress, SSS, SRS, SDS, and NRS were significant univariate predictors of critical events.

In the multivariate Cox proportional hazards model, age, hypertension, shortness of breath, extent of scarring (SRS), and extent of ischaemia (SDS) were independent predictors of events (Table 4). Hypercholesterolaemia and family history of CAD tended to be independent predictors of events. Of note, angina was not a significant predictor of critical events in this model, but shortness of breath was. In addition, a separate analysis was run in which the NRS was used as a surrogate marker for ejection fraction. NRS turned out to be a highly significant independent predictor of critical events (HR 1.16, 95\% Cl 1.06-1.26; $p=0.003$ ). 
Table 4 Results of the Cox proportional hazard's model for the prediction of critical events

\begin{tabular}{|c|c|c|c|c|c|}
\hline & \multirow[t]{2}{*}{ Hazard ratio } & \multirow[t]{2}{*}{$\chi^{2}$} & \multicolumn{2}{|c|}{ 95\% confidence interval } & \multirow[t]{2}{*}{$p$} \\
\hline & & & Lower & Upper & \\
\hline Age & 1.04 & 16.8 & 1.02 & 1.07 & $<0.0001$ \\
\hline Hypercholesterolaemia & 1.5 & 3.4 & 0.98 & 2.3 & 0.07 \\
\hline Family history of CAD & 1.6 & 3.5 & 0.98 & 2.5 & 0.06 \\
\hline Hypertension & 1.83 & 6.4 & 1.14 & 2.92 & 0.012 \\
\hline Shortness of breath & 2.1 & 7.8 & 1.24 & 3.42 & 0.005 \\
\hline SRS & 1.06 & 13.6 & 1.03 & 1.09 & $<0.0001$ \\
\hline SDS & 1.07 & 32.8 & 1.04 & 1.09 & $<0.0001$ \\
\hline
\end{tabular}

CE, critical event (myocardial infarction or cardiac death); SRS, summed rest score; SDS, summed difference score.

An increase in risk of $40 \%$ per decade of age was observed. The presence of hypertension increased risk by $83 \%$. Shortness of breath more than doubled risk. An increase of 10 points in SRS or SDS increased risk by $60 \%$ and $70 \%$, respectively.

After adjusting for historical and stress test data obtained before MPS, both scarring (SRS) and extent of ischaemia (SDS) remained significant predictors of critical events, thus yielding incremental value over prescan data. The incremental value of nuclear testing was reflected by a significant increase of global $\chi^{2}$ from 52 to 98 $(p<0.0001)$.

\section{Discussion}

This study of a large, consecutive series of diabetic patients referred for evaluation of possible or suspected CAD showed that silent CAD, as diagnosed by MPS, was found in $39 \%$ of patients. The rate of abnormal MPS results did not differ from that of patients with angina or anginalike chest pain, in contrast to nondiabetic patients. ${ }^{26}$ However, patients presenting with shortness of breath had a significantly higher rate of abnormal MPS results (higher incidence of CAD) than the other two groups. This finding underscores the fact that absence of angina is not equivalent to absence of CAD in diabetic patients.

If the study population was compared to a patient population with known CAD and stable angina, as in the Impact Of Nicorandil in Angina randomised trial (IONA), ${ }^{27}$ the diabetic patients of the present study without MPS evidence of CAD had an outcome similar to that of IONA study patients, with annual critical event rates of $1.9 \%$ and $2.9 \%$, respectively $(p=0.44)$. In contrast, the diabetic patients of the present study with MPS evidence of CAD had a significantly worse outcome than IONA study patients, with critical event rates of $5.4 \%$ and $2.9 \%$, respectively $(p<0.0001)$.

In the present study, in patients with normal MPS (no evidence of CAD) outcome did not differ in the three patient groups (asymptomatic patients, patients with angina, and patients with SOB). Furthermore, outcome was very similar in diabetic patients with MPS evidence of $C A D$, irrespective of the presence of chest pain. However, the outcome was significantly worse in patients with shortness of breath as their presenting symptom. Most likely this was due to the fact that these patients had MPS evidence of more prior silent myocardial infarctions and therefore had a significantly lower left ventricular ejection fraction than asymptomatic patients and patients with angina.

Age, hypertension, shortness of breath, and MPS variables of scarring and ischaemia were independent predictors of outcome. In addition, MPS provided incremental value to clinical and prescan variables to predict outcome in this relatively low-risk diabetic population.

\section{Incidence of silent CAD in diabetic patients}

In several studies evaluating the incidence of silent ischaemia in diabetic patients, the rate was as high as $65 \%$ and increased in various clinical settings and in the presence of risk factors. Higher rates were observed in patients older than 60 years ${ }^{28}$ and in patients with several risk factors, such as concomitant peripheral arterial vascular disease, ${ }^{7}$ retinopathy, ${ }^{6}$ microalbuminuria, ${ }^{10,29}$ and autonomic neuropathy. ${ }^{30}$

In a population-based autopsy study of decedents without clinical CAD, diabetes was associated with a global coronary artery disease burden and a prevalence of high-grade atherosclerosis similar to that observed among nondiabetic subjects with clinical CAD. ${ }^{31}$ Diabetic decedents (mean age $75 \pm 11$ years) and their nondiabetic counterparts (mean age $73 \pm 15$ years) had highgrade atherosclerosis in $68 \%$ and $46 \%$, respectively. ${ }^{31}$ Wackers et al. $^{32}$ recently reported the preliminary results of the Detection of Ischaemia in Asymptomatic Diabetics (DIAD) trial, the first large prospective trial evaluating silent CAD in diabetic patients. In a low-risk diabetic population (e.g., patients with ECG abnormalities consistent with CAD were not enrolled into the DIAD study), $27 \%$ of patients had evidence of abnormal MPS. These rates of silent CAD are lower than in the present study, where a higher risk group and older diabetic patients were studied.

\section{Impact of symptoms and silent CAD on prognosis in diabetic patients}

Several studies have shown that in the presence of myocardial ischaemia, diabetic patients report angina less frequently than nondiabetic patients ${ }^{20,33}$ and shortness 
of breath may be the only symptom of ischaemia. ${ }^{34}$ In the present study, only $45 \%$ of diabetic patients with MPS evidence of CAD had chest pain whereas $11 \%$ reported shortness of breath as the only symptom. In addition, the present findings show that outcome was related much more to the presence or absence of ischaemia and scarring than to symptoms: annual event rates were similar in patients with or without angina, whereas they were higher in diabetic patients presenting with shortness of breath. Thus, this symptom in diabetic patients seems to be related primarily to prior silent infarctions and not to ischaemia alone and should therefore be carefully considered in these patients.

There is only limited prognostic data of diabetic patients with silent ischaemia. In a relatively small patient population, Weiner et al. demonstrated that in the presence of exercise-induced myocardial ischaemia (whether silent or symptomatic), long-term survival was worse in diabetics than in nondiabetics. In contrast, in the absence of ischaemia, the mortality risk was not increased for diabetic patients. Importantly, survival rates among patients with silent ischaemia were similar to those of symptomatic patients regardless of diabetic status. ${ }^{33}$ In a scintigraphic study, diabetic patients with normal, mildly abnormal, and moderately to severely abnormal MPS results, assessed by the overall abnormality of MPS findings (SSS), had relatively low (1-2\% per year), intermediate (3-4\% per year), and high hard event rates $(>7 \%$ per year; $p<0.001)$, respectively. ${ }^{35}$ These findings were confirmed by Giri et al., ${ }^{36}$ who compared MPS results of diabetic versus nondiabetic patients with respect to prognosis. The presence and extent of abnormal MPS findings independently predicted subsequent cardiac events. Diabetic women had the worst outcome for any given extent of myocardial ischaemia. These results indicate that myocardial perfusion SPECT provides important incremental prognostic information in the evaluation of diabetic patients. The present study extends these observations to a large group of diabetic patients with a relatively low risk for CAD. Still, the observed event rates in asymptomatic patients were comparable to those of patients with angina, which underscores the relevance of silent CAD in diabetic patients and, hence, the importance of detecting silent CAD by MPS.

\section{Special aspects of the present study}

Besides objective evidence of ischaemia, scarring, and age, this study identified hypertension as an independent predictor of outcome in patients with diabetes. This is consistent with previous trials that demonstrated the outstanding importance of tight blood pressure control in diabetic patients. ${ }^{37-40}$ The hazard ratio in our Cox model indicated that the presence of hypertension had a similar impact on outcome, as a $12 \%$ or $14 \%$ increase in the extent of ischaemia or scarring, respectively. In addition, the present study underscores the importance of riskstratifying asymptomatic diabetic patients as recommended by the American Diabetes Association for high-risk patient subgroups. ${ }^{41}$ However, these recom- mendations were based on the clinical judgment of a panel of experts rather than on published data. ${ }^{42}$ Our study adds evidence to these recommendations and suggests a role for cardiac imaging in diabetic patients. Finally, results of noninvasive testing may become relevant for treatment decisions, although specific trials in diabetic patients are lacking. Still, the findings of The Asymptomatic Cardiac Ischemia Pilot Study (ACIP) showed that in patients with silent ischaemia, revascularisation significantly reduced death, myocardial infarction, and cardiac hospitalisation compared to such patients managed conservatively. ${ }^{43}$

\section{Limitations}

Regarding the incidence of silent ischaemia in diabetic patients, there is a certain bias in our patient population because patients were referred for testing based on clinical circumstances. There is also no detailed information about the type and duration of diabetes, mode of treatment, or degree of diabetic control, and only insufficient information was available about secondary organ involvement and autonomic nerval dysfunction, variables that have been shown to be associated with a higher rate of CAD in diabetic patients. However, the present study focused on symptomatic presentation in diabetic patients and its relation to MPS findings and outcome rather than on degree of diabetes control.

\section{Conclusions}

In an asymptomatic, diabetic patient population referred for evaluation of $C A D$, the frequency of scintigraphic evidence of CAD was $39 \%$, as high as in patients with angina. Accordingly, the annual critical event rate was similarly and strongly dependent on objective evidence of CAD. The proportion of patients with MPS evidence of CAD was significantly higher in patients presenting with shortness of breath and outcome was more than three times worse, most likely due to prior silent infarctions, as shown by larger areas of scarring and a worse left ventricular ejection fraction. Age and hypertension were other independent predictors of events. MPS added incremental information to clinical and prescan information to predict outcome, indicating that MPS seems to be valuable for risk stratification of asymptomatic diabetic patients with respect to the diagnosis of CAD and its prognosis.

\section{References}

1. Zimmet $P$, Alberti KG, Shaw J. Global and societal implications of the diabetes epidemic. Nature 2001;414:782-7.

2. Zimmet PZ, McCarty DJ, de Courten MP. The global epidemiology of non-insulin-dependent diabetes mellitus and the metabolic syndrome. J Diabetes Complications 1997;11:60-8. 
3. Grundy SM, Benjamin IJ, Burke GL et al. Diabetes and cardiovascular disease: a statement for healthcare professionals from the American Heart Association. Circulation 1999;100:1134-46.

4. Kannel WB, McGee DL. Diabetes and cardiovascular disease. The Framingham study. JAMA 1979;241:2035-8.

5. Feit F, Brooks MM, Sopko G et al. Long-term clinical outcome in the Bypass Angioplasty Revascularization Investigation Registry: comparison with the randomized trial. BARI Investigators. Circula tion 2000;101:2795-802.

6. Janand-Delenne B, Savin B, Habib G et al. Silent myocardial ischemia in patients with diabetes: who to screen. Diabetes Care 1999;22:1396-400.

7. Nesto RW, Watson FS, Kowalchuk GJ et al. Silent myocardial ischemia and infarction in diabetics with peripheral vascular disease: assessment by dipyridamole thallium-201 scintigraphy. Am Heart $J$ 1990;120:1073-7.

8. Nesto RW, Phillips RT. Asymptomatic myocardial ischemia in diabetic patients. Am J Med 1986;80:40-7.

9. Zellweger MJ, Pfisterer ME. Silent coronary artery disease in patients with diabetes mellitus. Swiss Med Wkly 2001;131:427-32.

10. Rutter MK, Wahid ST, McComb JM et al. Significance of silent ischemia and microalbuminuria in predicting coronary events in asymptomatic patients with type 2 diabetes. J Am Coll Cardiol 2002;40:56-61.

11. Hachamovitch R, Berman DS, Shaw LJ et al. Incremental prognostic value of myocardial perfusion single photon emission computed tomography for the prediction of cardiac death: differential stratification for risk of cardiac death and myocardial infarction. Circulation 1998;97:535-43.

12. Hachamovitch $\mathrm{R}$, Berman DS, Kiat $\mathrm{H}$ et al. Effective risk stratification using exercise myocardial perfusion SPECT in women: gender-related differences in prognostic nuclear testing. $J$ Am Coll Cardiol 1996;28:34-44.

13. Hachamovitch $\mathrm{R}$, Berman DS, Kiat $\mathrm{H}$ et al. Exercise myocardial perfusion SPECT in patients without known coronary artery disease: incremental prognostic value and use in risk stratification. Circulation 1996;93:905-14.

14. Hachamovitch R, Berman DS, Kiat $\mathrm{H}$ et al. Value of stress myocardial perfusion single photon emission computed tomography in patients with normal resting electrocardiograms: an evaluation of incremental prognostic value and cost-effectiveness. Circulation 2002; 105:823-9.

15. Zellweger MJ, Dubois EA, Lai $S$ et al. Risk stratification in patients with remote prior myocardial infarction using rest-stress myocardial perfusion SPECT: prognostic value and impact on referral to early catheterization. J Nucl Cardiol 2002;9:23-32.

16. Zellweger MJ, Lewin HC, Lai S et al. When to stress patients after coronary artery bypass surgery? Risk stratification in patients early and late post-CABG using stress myocardial perfusion SPECT: implications of appropriate clinical strategies. J Am Coll Cardiol 2001;37:144-52.

17. Berman DS, Kiat H, Friedman JD et al. Clinical applications of exercise nuclear cardiology studies in the era of healthcare reform. Am J Cardiol 1995;75:3D-13D.

18. Berman DS, Germano G, Shaw LJ. The role of nuclear cardiology in clinical decision making. Semin Nucl Med 1999;29:280-97.

19. Zellweger MJ, Weinbacher M, Zutter AW et al. Long-term outcome of patients with silent versus symptomatic ischemia six months after percutaneous coronary intervention and stenting. J Am Coll Cardiol 2003;42:33-40.

20. Nesto RW, Phillips RT, Kett KG et al. Angina and exertional myocardial ischemia in diabetic and nondiabetic patients: assessment by exercise thallium scintigraphy. Ann Intern Med 1988; 108:170-5.

21. Nesto RW. Screening for asymptomatic coronary artery disease in diabetes. Diabetes Care 1999;22:1393-5.

22. Ladenheim ML, Pollock BH, Rozanski A et al. Extent and severity of myocardial hypoperfusion as predictors of prognosis in patients with suspected coronary artery disease. J Am Coll Cardiol 1986; 7:464-71.

23. Staniloff HM, Forrester JS, Berman DS et al. Prediction of death, myocardial infarction, and worsening chest pain using thallium scintigraphy and exercise electrocardiography. $J$ Nucl Med 1986;27:1842-8.

24. Berman DS, Kiat $H$, Friedman JD et al. Separate acquisition rest thallium-201/stress technetium-99m sestamibi dual-isotope myocardial perfusion single-photon emission computed tomography: a clinical validation study. J Am Coll Cardiol 1993;22: 1455-64.

25. Berman DS, Hachamovitch $\mathrm{R}$, Kiat $\mathrm{H}$ et al. Incremental value of prognostic testing in patients with known or suspected ischemic heart disease: a basis for optimal utilization of exercise technetium$99 \mathrm{~m}$ sestamibi myocardial perfusion single-photon emission computed tomography. J Am Coll Cardiol 1995;26:639-47.

26. Udelson JE, Rajendran V, Leppo JA. Diagnosis of coronary artery disease. In: Murray IPC, Ell PJ, editors. Nuclear medicine in clinical diagnosis and treatment. 2nd ed. Churchill Livingstone; 1998. p. 1389-413.

27. Effect of nicorandil on coronary events in patients with stable angina: the Impact Of Nicorandil in Angina (IONA) randomised trial. Lancet 2002;359:1269-75.

28. Inoguchi $\mathrm{T}$, Yamashita $\mathrm{T}$, Umeda $\mathrm{F}$ et al. High incidence of silent myocardial ischemia in elderly patients with non insulin-dependent diabetes mellitus. Diabetes Res Clin Pract 2000;47:37-44.

29. Rutter MK, McComb JM, Brady S et al. Silent myocardial ischemia and microalbuminuria in asymptomatic subjects with non-insulin-dependent diabetes mellitus. Am J Cardiol 1999;83:27-31.

30. Langer A, Freeman MR, Josse RG et al. Detection of silent myocardial ischemia in diabetes mellitus. Am J Cardiol 1991;67:1073-8.

31. Goraya TY, Leibson CL, Palumbo PJ et al. Coronary atherosclerosis in diabetes mellitus: a population-based autopsy study. J Am Coll Cardiol 2002;40:946-53.

32. Wackers FJ, Young LH, Inzucchi SE et al. Detection of ischemia in asymptomatic diabetics: preliminary results of the DIAD study. J Am Coll Cardiol 2003;41:409.

33. Weiner DA, Ryan TJ, Parsons $L$ et al. Significance of silent myocardial ischemia during exercise testing in patients with diabetes mellitus: a report from the Coronary Artery Surgery Study (CASS) Registry. Am J Cardiol 1991;68:729-34.

34. Margolis JR, Kannel WS, Feinleib $M$ et al. Clinical features of unrecognized myocardial infarction - silent and symptomatic. Eighteen year follow-up: the Framingham study. Am J Cardiol 1973; 32:1-7

35. Kang X, Berman DS, Lewin HC et al. Incremental prognostic value of myocardial perfusion single photon emission computed tomography in patients with diabetes mellitus. Am Heart $J$ 1999;138:102532.

36. Giri S, Shaw LJ, Murthy DR et al. Impact of diabetes on the risk stratification using stress single-photon emission computed tomography myocardial perfusion imaging in patients with symptoms suggestive of coronary artery disease. Circulation 2002;105:3240.

37. Grossman E, Goldbourt U. Hypertension Optimal Treatment (HOT) trial. Lancet 1998;352:572, discussion 574-5.

38. Klovning A, Sandvik H, Straand J. The HOT study. Can increased therapeutic intensity be dangerous? Tidsskr Nor Laegeforen 1998;118:4405-6.

39. Efficacy of atenolol and captopril in reducing risk of macrovascular and microvascular complications in type 2 diabetes: UKPDS 39. UK Prospective Diabetes Study Group. BMJ 1998;317:713-20.

40. Effects of ramipril on cardiovascular and microvascular outcomes in people with diabetes mellitus: results of the HOPE study and MICROHOPE substudy. Heart Outcomes Prevention Evaluation Study Investigators. Lancet 2000;355:253-9.

41. ADA. Consensus development conference on the diagnosis of coronary heart disease in people with diabetes: 10-11 February 1998, Miami, Florida. American Diabetes Association. Diabetes Care 1998;21:1551-9.

42. Wackers FJ, Zaret BL. Detection of myocardial ischemia in patients with diabetes mellitus. Circulation 2002;105:5-7.

43. Rogers WJ, Bourassa MG, Andrews TC et al. Asymptomatic Cardiac Ischemia Pilot (ACIP) study: outcome at 1 year for patients with asymptomatic cardiac ischemia randomized to medical therapy or revascularization. The ACIP Investigators. J Am Coll Cardiol 1995;26:594-605. 